\title{
Automated fabrication of hybrid thermoplastic prepreg material to be processed by In-Situ Consolidation Automated Fiber Placement process
}

\author{
Vincenzo Iannone ${ }^{*}$,Marco Barile ${ }^{1}$, Leonardo Lecce ${ }^{1}$ \\ ${ }^{1}$ NOVOTECH Aerospace Advanced Technology Srl, 80125 Piazzale D'Annunzio 15, Naples, Italy
}

\begin{abstract}
This work deals with the fabrication of an innovative hybrid thermoplastic prepreg by continuous hot forming process. The material, suitable also for Automated Fiber Placement process, is produced through a consolidation of commercial PEEK-Carbon Fiber prepreg sandwiched between two amorphous PEI films. Consolidation is performed by a purpose-designed automated prototype equipment operating on defined pressure and thermal cycles. Then preliminary tests on first trials produced were carried out. These activities have been developed in the frame of the NHYTE project, a Research and Innovation Action funded by the European Union's H2020 framework programme, under Grant Agreement No 723309 NOVOTECH acting as Coordinator presents this paper on behalf of all Partners of the project. The proof of NHYTE project concept is the manufacturing of a fastener free and high performing fuselage portion demonstrator.
\end{abstract}

\section{Introduction}

\subsection{State-of-the-Art}

The PEEK-Carbon Fiber prepreg is a widely used material for commercial applications and properties of the structures realized with this material are similar to the ones performed with the best class of thermosets, with some specific properties (e.g. toughness and impact resistance) better than those of the best thermosets. Such prepreg ensures both functions of toughness enhancement (multilayer material) and process simplification, including improved cycle times and lower energy consumptions, since it does not require the use of an autoclave curing phase.

In order to be used for aircraft structures, they need to be processed above the melting temperature of the crystalline phase of PEEK (at least $380{ }^{\circ} \mathrm{C}$ ). Furthermore the cooling rate must be kept inside a fixed processing window because a too fast cooling induces a decrease of crystallinity content while a too slow cooling induces a crystallinity increase, compared with the standard one [1]. All these aspects make APC-2 processing expensive,

*Corresponding author: vincenzo.iannone@novotech.it 
and the full exploitation of the potentially obtained advanges of using the thermoplastic matrix composites is hindered.

\subsection{Proposed Innovation}

An innovative solution is proposed based on the addition of amorphous PEI films to Carbon-PEEK prepreg, performed in condition of controlled temperature history. That allows to perform a composite with PEEK at the desired crystallinity level layered between amorphous films. The adhesion between PEEK and PEI in the hybrid material is very good thanks to PEEK and PEI deep compatibility and to their attitude to produce blends when mixed. For the same reason the addition of PEI to the PEEK-carbon fiber prepreg doesn't affect the recyclability, because PEEK, PEI and PEEK-PEI blends are recyclable at the same level. Starting from the state-of-art of the hybrid thermoplastic material, conceived and patented by LEONARDO S.p.A, a partner of the NHYTE Consortium [2], NOVOTECH is currently developing an accurate study aimed to identify critical process parameters (e.g. temperature, pressure, time, feeding speed rate) and their range of variation in order to set the best settings of the automated prototype equipment/cell to use for such new hybrid thermoplastic material fabrication.

\section{Process Description}

The hybrid prepreg fabrication process is based on heating the PEEK-carbon prepreg and the additional PEI films in a condition in which both are fluid, e.g. above the temperature of crystalline melting of PEEK $\mathrm{T}_{\mathrm{m}}\left(343{ }^{\circ} \mathrm{C}\right)$, consolidating the prepreg under pressure, and cooling the obtained prepreg under controlled conditions.

The process being optimized in Novotech uses a press with plates at different temperatures. The control of thermal history is performed by setting the temperatures of the plates and the motion of the processed hybrid prepreg under the press. The prepreg movement is obtained by opening the press, shifting the assembly of the prepreg and the additional amorphous films in the press length direction, and closing the press again. With this method the different sections of the prepreg change progressively the temperature, but the press temperature is kept constant in the different sections. That allows to perform a process that is conceptually continuous, and more affordable by the energy point of view (no press cooling and heating required).

The motion of the hybrid prepreg under the press is sketched below from Figure 1(a) to Figure 1(d).

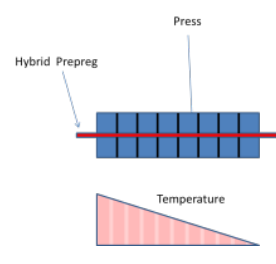

(a) Closed Press

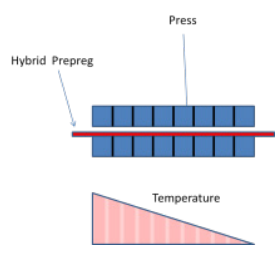

(b) Press Opening

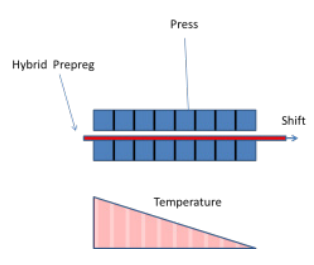

(c) Prepreg Shifting

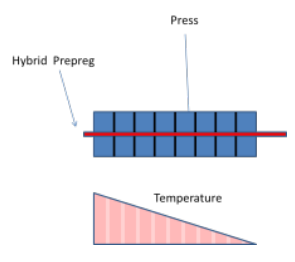

(d) Press Closing

Figure 1 Hybrid Prepreg Motion under the Press 
The change of temperature of the assembly moving through the press is reported below in Figure 2 for a press with different temperature zones.

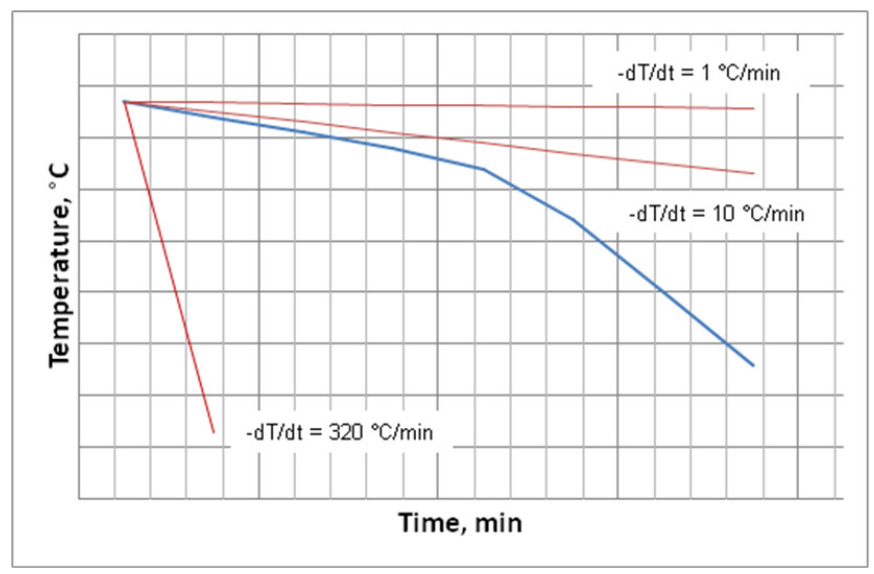

Figure 2 Qualitative temperature history of the processed prepreg under the press

The temperature distribution of the plates must be optimized taking in account several factors. The temperature in the hot zone must allow the consolidation of lay-up, including correct bonding between PEEK and PEI. For this reason the first zone is above the temperature of melting of the crystalline phase of PEEK $\left(343^{\circ} \mathrm{C}\right)$ and both PEEK and PEI are liquid. In this situation there is a zone in which PEEK and PEI are interpenetrated, forming a transition zone from $100 \%$ of PEEK to $100 \%$ of PEI, with variable percentage; in the zone with both PEEK and PEI blends are obtained, characterized by a crystalline phase which is the PEEK standard one, with a percentage depending on the PEEK amount, and a blended amorphous phase, with a $\mathrm{T}_{\mathrm{g}}$ intermediate between the $\mathrm{T}_{\mathrm{g}}$ of PEEK and PEI. Cooling to the crystalline melting temperature, a crystalline zone is created. The control of cooling rate in this phase is very important, because the right level of crystallinity and the right structure of the crystals is obtained only if cooling rate falls in a processing window from 1 to $320^{\circ} \mathrm{C} / \mathrm{min}$. These max/min cooling rates, together with a typical cooling rate of $10{ }^{\circ} \mathrm{C} / \mathrm{min}$ are reported in Figure 2. The processing window is very wide, but a passage from a plate to another could give a fast cooling; this is the reason why the temperature difference is reduced in the zone of Tm. Considering the temperature of the hybrid prepreg as equal to the one of the press plate is correct unless the approximations depending on:

- change of the temperature through the thickness; this change is limited, due to the small thickness, but must be considered in a detailed calculation;

- time of open press; during this period (Figure 1(b)-(c)) the lower side of the assembly is still in contact with the lower plate of the press, but the upper side is exposed to the open environment. Calculation of the convective thermal exchange isn't trivial, because the air temperature between the plates is lower than the one of plates, but will be higher than room temperature. On the other hand the lower side of the assembly is always in contact with the press, and it's correct to suppose that its lower side surface temperature is equal to the press one.

\section{Produced Material Evaluation}


The temperature distribution in the hybrid material during processing is being modelled during the project, but a direct control on the suitability of the obtained cooling rate can be performed through crystallinity evaluation by DSC. In fact, in Figure 3 a typical differential scanning calorimetry plot of PEEK is shown.

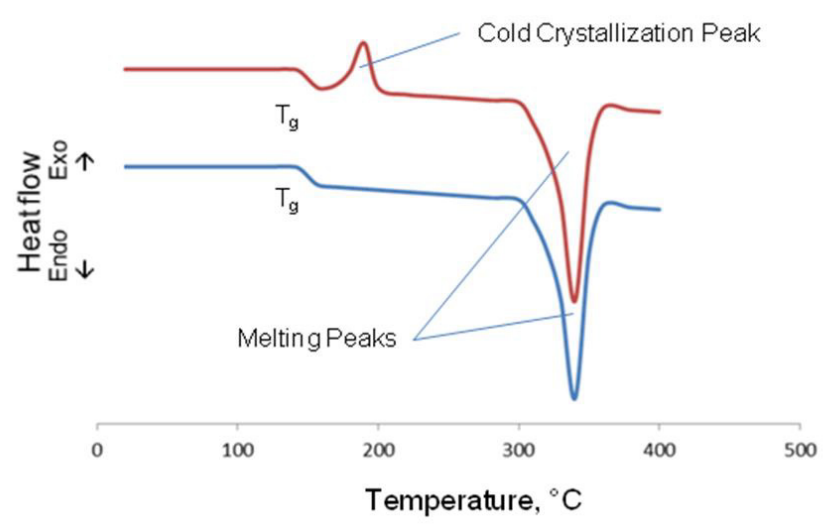

Figure 3 Typical DSC scans of PEEK, semi-crystalline and amorphous

The lower scan (blue curve) reported in figure is representative of PEEK cooled within the processing window mentioned above. In this line can be observed the glass transition zone, related to the molecular motion of the amorphous phase, and a melting peak, depending on the heat amount needed to melt the crystalline phase.

The upper scan (red curve) is representative of PEEK partially crystallized (or completely amorphous); during the heating above the $\mathrm{T}_{\mathrm{g}}$ a crystallization happens, at a temperature below the $T_{m}$, due to the instability of the amorphous phase. When the melting temperature is reached both crystals melt, the one formed by cold crystallization and the ones formed during DSC heating. The difference between the two peak areas allows us to evaluate the sample crystallinity.

In the hybrid fabrication process optimization DSC is being used to check that cooling rate is in the right window.

Pressure must be sufficient to obtain consolidation of PEI films on the PEEK-carbon prepregs, but an excess of flow is unwanted. The optimization of pressure must be performed together with the one of thermal history, which controls both crystallinity (as previously described) and viscosity. Different production runs are being performed with a design of experiment (DOE) approach, and the quality of the obtained samples is being evaluated through measurement of thickness and areal weight.

The first test trial produced is a $200 \times 200 \mathrm{~mm}$ flat panel with 4 plies of hybrid TP (PEI/APC-2 PEEK/PEI) and the stacking sequence is $\left[0^{\circ} / 90^{\circ} / 90^{\circ} / 0^{\circ}\right]$. Sample data are reported in the following Table 1.

Visual inspection of the specimen shows no defects or consolidation anomalies on both top and bottom surfaces. Microscopic qualitative view (Figure 4) doesn't show any porosity or delamination. 


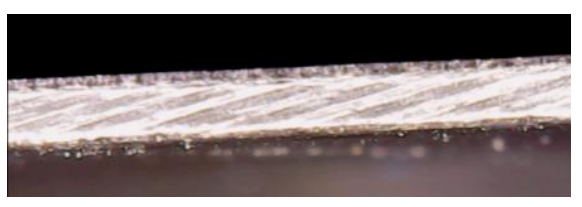

Figure 4 Microscopic Qualitative View

Table 1 Sample Data

\begin{tabular}{|c|c|}
\hline Resin Content (APC2-PEEK) & $34 \%$ \\
\hline Areal Weight (APC2-PEEK) & $145 \mathrm{~g} / \mathrm{m} 2$ \\
\hline Thickness (APC2-PEEK) & $0.14 \mathrm{~mm}$ \\
\hline Density (PEI) & $1.27 \mathrm{~g} / \mathrm{m} 3$ \\
\hline Thickness (PEI) & $0.05 \mathrm{~mm}$ \\
\hline
\end{tabular}

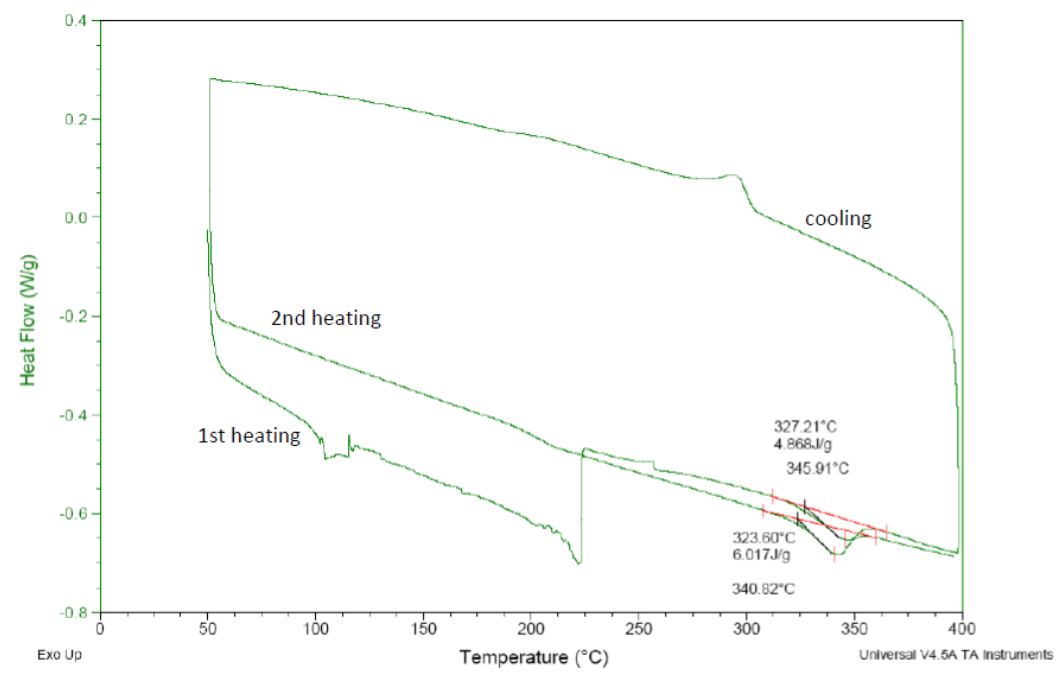

Figure 5 DSC scanning of the first trial produced with hybrid TP

The DSC scanning performed by CETMA shows the thermal behavior of the sample analyzed. The scan consist of three steps, heat/cool/heat from $25^{\circ} \mathrm{C}$ to $400^{\circ} \mathrm{C}$ (test conditions reported in Tab) with a heat rate of $10^{\circ} \mathrm{C} / \mathrm{min}$. The signal of first heating is slightly disturbed maybe due to the presence of some impurities or maybe to measurement errors occurred. The most remarkable event is the melting of the PEEK matrix, visible with the endothermal signal that starts at a temperature of about $320^{\circ} \mathrm{C}$. The peak temperature is about $345^{\circ} \mathrm{C}$ and the heat of fusion is calculated by the area under the base line normalized with respect to the sample weight. The same calculation is done for the second heating, in which is not observed the previous disturbance. The first heating gives information about the productive process so the crystallinity percentage calculated is the really reached one for this material with these specific process parameters. Instead, the crystallinity percentage 
calculated in the second heating is the maximum achievable, because all of its previous thermal history has been deleted by the first heating. No cold crystallization peak has been detected.

Table 2 Test Data

\begin{tabular}{|c|c|}
\hline Differential Scanning Calorimeter & Mettler Toledo Star ${ }^{\mathrm{e}}$ System DSC823 \\
\hline Balance & Analytical RADWAG XA $60 / 220$ \\
\hline Thermal Cycle & Heat/Cool/Heat $\left(25-400{ }^{\circ} \mathrm{C} / \mathrm{min}\right)$ \\
\hline Material of the specimen capsule & Aluminium \\
\hline Purge Gas & Nitrogen \\
\hline Purge Gas Flow & $80 \mathrm{~mL} / \mathrm{min}$ \\
\hline Purge Gas Purity & $95-98 \%$ \\
\hline
\end{tabular}

The percent of crystallinity $(P C)$ reported below in Table 3 has been computed as follows:

$$
P C=\left(\Delta H_{f}-\Delta H_{c c}\right) /\left(\Delta H_{f}^{0} \cdot R_{C}\right)
$$

where $\Delta H_{f}$ is the heat of fusion (first heat cycle), $\Delta H_{c c}$ is the heat of cold crystallization, $\Delta H_{f}^{0}$ is the theoretical maximum heat of fusion of a fully crystalline sample and $R_{C}$ is the resin content (in percent) for the prepreg material.

Table 3 Results

\begin{tabular}{|l|l|}
\hline Percent Crystallinity (1st heat cycle) & $17.40 \%$ \\
\hline Percent Crystallinity (2nd heat cycle) & $21.50 \%$ \\
\hline
\end{tabular}

\section{Conclusion}

Research is currently in progress but first DSC analysis gave encouraging results in terms of percent cristallinity and indicates a good physical and chemical quality of the hybrid TP lamina produced, with positive indications in terms of feasibility of the hybrid semicrystalline-amorphous prepreg investigated in this research project. This first result ensures that through the accurate refinement of process parameters it will be also possible to significantly increase percent crystallinity of the hybrid prepreg lamina up to $5-8 \%$.

A large manufacturing campaign, according to the DOE methodology, is under development in order to collect further data about:

- lamina thicknesses distribution;

- areal weights distribution;

- correlation of the hybrid prepreg physical properties (DSC) vs. processing parameters (also at different PEI thicknesses);

- Ultrasonic NDI analysis. 
A special thanks goes to all the partners involved in the NHYTE project for their hiprofile scientific work and in particular to CETMA for the prompt and detailed DSC results that have been of crucial importance for the results of the present paper.

\section{References}

1. J. Kenny, A. D'Amore, L. Nicolais, M. Iannone, B. Scatteia, SAMPE Journal Vol. 25, 4, (1989)

2. Preimpregnated materials with semi-crystalline matrix and amorphous surface layers EP 2109532 B1 of 02/03/2011, LEONARDO S.p.A., (2011)

3. Lustiger A, Uralil FS, Newaz GM. Polym Compos;11:65-75 (1990) 